\title{
Switching of Intramolecular Charge Transfer in Cruciforms: Metal Ion Sensing.
}

\author{
James N. Wilson and Uwe H. F. Bunz* \\ School of Chemistry and Biochemistry, Georgia Institute of Technology, \\ 770 State St., Atlanta, GA 30332,USA. \\ uwe.bunz@chemistry.gatech.edu
}
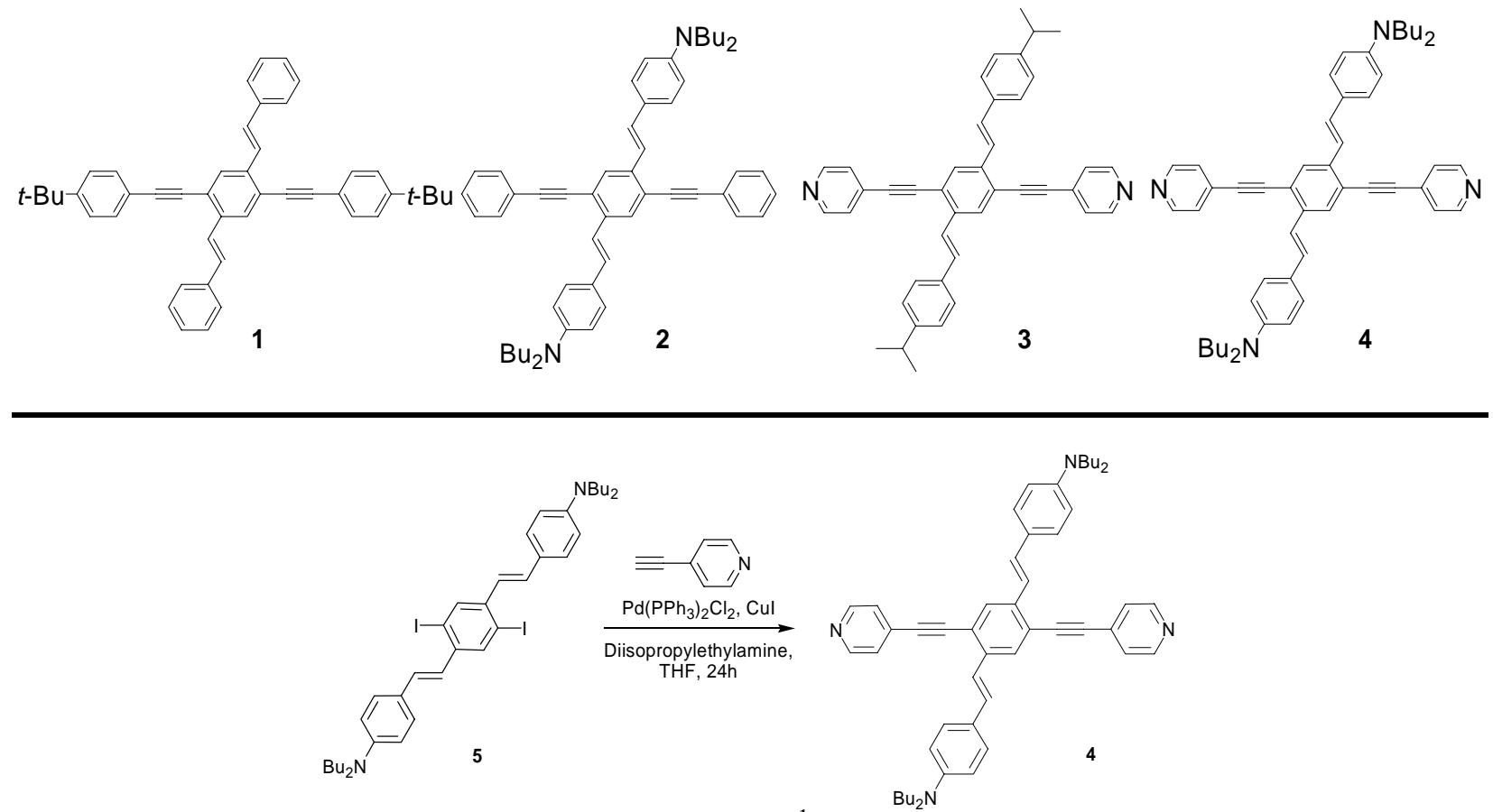

Cruciform 4: Compound 5 (560 mg, $0.758 \mathrm{mmol})^{1}$ was combined with 4-ethynylpyridine (300 $\mathrm{mg}, 2.91 \mathrm{mmol}),\left(\mathrm{PPh}_{3}\right)_{2} \mathrm{PdCl}_{2}(5.0 \mathrm{mg}, 7.1 \mu \mathrm{mol}), \mathrm{CuI}(5.0 \mathrm{mg}, 33 \mu \mathrm{mol}), 2 \mathrm{~mL}$ of Hünig's base, and $2 \mathrm{~mL}$ of THF in a Schlenk tube under nitrogen purge. The reaction was capped with a septum, placed in a warm water bath $\left(45^{\circ} \mathrm{C}\right)$, and allowed to react for $24 \mathrm{~h}$. The crude reaction mixture was poured into a $1 \mathrm{~L}$ flask containing $250 \mathrm{~mL}$ of $5 \%$ aqueous $\mathrm{NH}_{4} \mathrm{OH}$ and $250 \mathrm{~mL}$ of hexane. The mixture was vigorously stirred, then filtered, isolating a bright orange solid. The solid was collected, dissolved in a minimum volume of dichloromethane and precipitated into $250 \mathrm{~mL}$ of rapidly stirring hexane. The precipitate was collected yielding $347 \mathrm{mg}$ of bright orange crystals after careful drying under oil pump vacuum. An analytically pure sample was obtained by crystallization of 4 from dichloromethane. Yield: 62\%; M.p.: 201-203 ${ }^{\circ}$ C. IR: v $\left(\mathrm{cm}^{-1}\right)$ 2956, 2869, 1600, 1589, 1517, 1399, 1366, 1313, 1281, 1218, 1184, 1151, 955, 821, 804. ${ }^{1} \mathrm{H}$ NMR $\left(300 \mathrm{MHz}, \mathrm{CDCl}_{3}\right): \delta=8.66(\mathrm{bs}, 4 \mathrm{H}), 7.87(\mathrm{~s}, 2 \mathrm{H}), 7.46-7.41(\mathrm{~m}, 8 \mathrm{H}), 7.35(\mathrm{~d}, 2 \mathrm{H}$, $\left.\mathrm{J}_{3 \mathrm{H}, \mathrm{H}}=16.2 \mathrm{~Hz}\right), 7.18\left(\mathrm{~d}, 2 \mathrm{H}, \mathrm{J}_{3 \mathrm{H}, \mathrm{H}}=16.2 \mathrm{~Hz}\right), 6.65(\mathrm{bd}, 4 \mathrm{H}), 3.32\left(\mathrm{t}, 8 \mathrm{H}, \mathrm{J}_{3 \mathrm{H}, \mathrm{H}}=7.7 \mathrm{~Hz}\right), 1.75-$ $1.52(\mathrm{~m}, 8 \mathrm{H}), 1.38-1.33(\mathrm{~m}, 8 \mathrm{H}), 0.96\left(\mathrm{t}, 12 \mathrm{H}, \mathrm{J}_{3 \mathrm{H}, \mathrm{H}}=7.4 \mathrm{~Hz}\right) .{ }^{13} \mathrm{C} \mathrm{NMR}\left(75.5 \mathrm{MHz}, \mathrm{CDCl}_{3}\right): \delta$ $=149.95,148.31,137.85,131.63,128.65,125.74,124.34,121.14,119.92,111.86,93.24,92.51$, 51.15, 29.85, 20.75, 14.45. Anal. Calcd.: C, 84.51; H, 7.91. Found: C, 84.33; H, 7.78. 


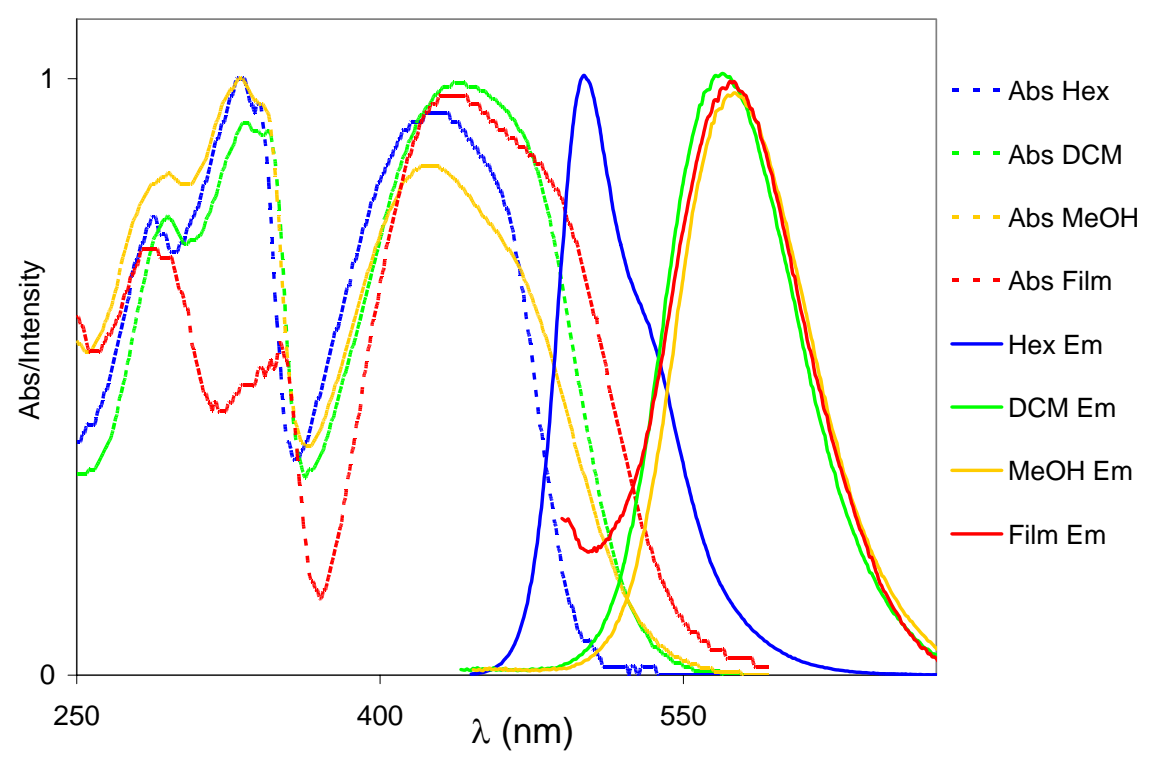

Figure 1. Absorption and emission spectra of $\mathbf{4}$ under different conditions.

Table 1. Summary of optical data for compound 4.

\begin{tabular}{|c|c|c|c|c|}
\hline & Hexane & DCM & MeOH & Thin film \\
\hline $\begin{array}{c}\text { Absorbance } \\
\lambda_{\max }(\mathrm{nm})\end{array}$ & 331,429 & 335,440 & 332,435 & 437 \\
\hline & & $\varepsilon=53,800 \mathrm{~cm}^{-1}$ & & \\
\hline $\begin{array}{c}\text { Emission } \\
\lambda_{\max }(\mathrm{nm})\end{array}$ & 502 & 571 & 576 & 576 \\
\hline & & & & \\
\hline
\end{tabular}

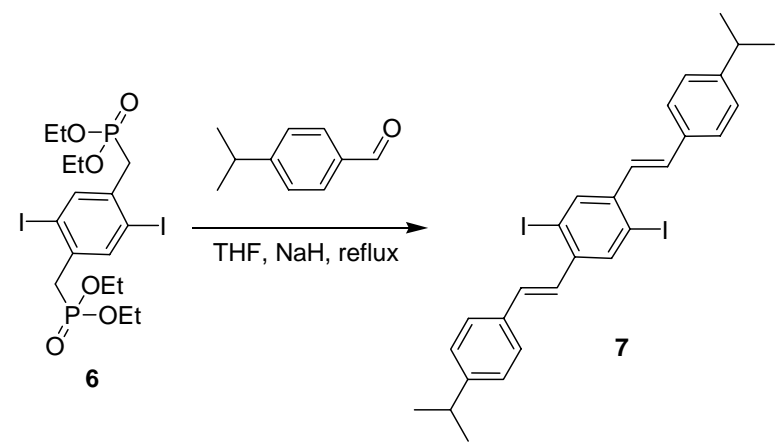

Compound 7: $\mathbf{6}^{2}(2.00 \mathrm{~g}, 3.17 \mathrm{mmol})$ was placed in a $500 \mathrm{~mL}$ 2-neck-flask equipped with a stirbar under nitrogen purge; $150 \mathrm{~mL}$ of dry THF were added, followed by $\mathrm{NaH}(0.190 \mathrm{~g}, 7.90 \mathrm{mmol})$. A condenser was placed atop the reaction vessel, capped by a balloon. The $2^{\text {nd }}$ neck was capped with a septum and secured by copper wire. The reaction was heated to mild reflux and 4isopropylbenzaldehyde (1.18 $\mathrm{g}, 7.90 \mathrm{mmol})$ was introduced in $0.1 \mathrm{~mL}$ aliquots over a period of 30 min. The reaction mixture was cooled to room temperature and placed in an ice bath. The excess $\mathrm{NaH}$ was quenched with slow addition of $\mathrm{MeOH} ; 200 \mathrm{~mL}$ of hexane and $100 \mathrm{~mL}$ of water were added to the reaction mixture. The crude mixture was filtered rinsed with $\mathrm{MeOH}$. The bright yellow-green crystals were dissolved in $50 \mathrm{~mL}$ of dichloromethane and precipitated into $300 \mathrm{~mL}$ of hexane yielding $1.54 \mathrm{~g}$ of bright-yellow green crystals after removal of the 
solvent and drying on an oil vacuum pump. Yield: $79 \%$. MP: 205-206 . IR: $v\left(\mathrm{~cm}^{-1}\right) 3004$, 2950, 2929, 2856, 1628, 1512, 1454, 1355, 1329, 1243, 1052, 1041, 962, 890, 864, 854, 824, 816. ${ }^{1} \mathrm{H}$ NMR $\left(300 \mathrm{MHz}, \mathrm{CDCl}_{3}\right): \delta=8.06(\mathrm{~s}, 2 \mathrm{H}), 7.49\left(\mathrm{~d}, \mathrm{~J}_{3 \mathrm{H}, \mathrm{H}}=8.2 \mathrm{~Hz}, 4 \mathrm{H}\right), 7.25\left(\mathrm{~d}, \mathrm{~J}_{3 \mathrm{H}, \mathrm{H}}\right.$ $=8.2 \mathrm{~Hz}, 4 \mathrm{H}), 7.15\left(\mathrm{~d}, \mathrm{~J}_{3 \mathrm{H}, \mathrm{H}}=16.2 \mathrm{~Hz}, 2 \mathrm{H}\right), 6.96\left(\mathrm{~d}, \mathrm{~J}_{3 \mathrm{H}, \mathrm{H}}=16.2 \mathrm{~Hz}, 2 \mathrm{H}\right), 3.01-2.87(\mathrm{~m}, 2 \mathrm{H})$, $1.28\left(\mathrm{~d}, \mathrm{~J}_{3 \mathrm{H}, \mathrm{H}}=6.87 \mathrm{~Hz}, 12 \mathrm{H}\right) .{ }^{13} \mathrm{C}$ NMR $\left(75.5 \mathrm{MHz}, \mathrm{CDCl}_{3}\right): \delta=149.52,140.87,136.30$, $134.43,132.34,129.84,127.23,127.10,100.65,34.35,24.32$.

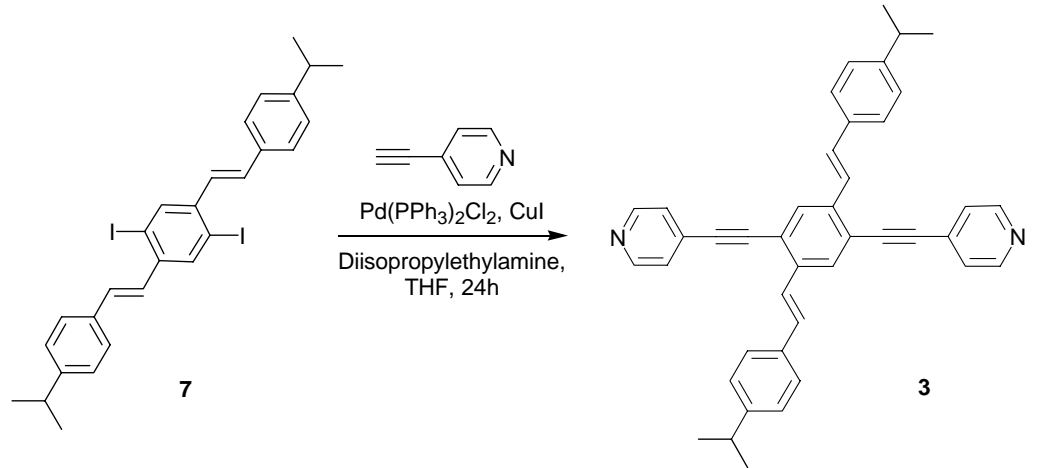

Compound 3: 7 (500 mg, 0.809 mmol) was combined with 4ethynylpyridine $(209 \mathrm{mg}, 2.02$ $\mathrm{mmol}),\left(\mathrm{PPh}_{3}\right)_{2} \mathrm{PdCl}_{2}(5.0 \mathrm{mg}, 7.1$ $\mu \mathrm{mol}), \mathrm{CuI}(5.0 \mathrm{mg}, 33 \mu \mathrm{mol}), 2$ $\mathrm{mL}$ of Hünig's base, and $2 \mathrm{~mL}$ of THF in a Schlenk tube under nitrogen purge. The reaction vessel was capped with a septum, placed in a warm water bath

$\left(45^{\circ} \mathrm{C}\right)$, and allowed to react for $24 \mathrm{~h}$. The crude reaction mixture was poured into a $1 \mathrm{~L}$ flask containing $250 \mathrm{~mL}$ of $5 \%$ aqueous $\mathrm{NH}_{4} \mathrm{OH}$ and $250 \mathrm{~mL}$ of hexane. The mixture was vigorously stirred, then filtered isolating a bright yellow solid. The solid was collected, dissolved in a minimum volume of dichloromethane and precipitated into $250 \mathrm{~mL}$ of rapidly stirring hexane. The precipitate was collected yielding $320 \mathrm{mg}$ of yellow crystals after carefully drying in oil pump vacuum at $40^{\circ} \mathrm{C}$. An analytically pure sample was obtained by crystallization of 3 from dichloromethane. Yield: 70\%; M.p.: 273-275 $\mathrm{C}^{\mathrm{O}}(\mathrm{dec})$. IR: $v\left(\mathrm{~cm}^{-1}\right) 3044,3023,2957,2869$, $1591,1538,1514,1458,1404,1249,1214,967,864,817,625 .{ }^{1} \mathrm{H}$ NMR $\left(300 \mathrm{MHz}, \mathrm{CDCl}_{3}\right): \oint$ $=8.67(\mathrm{bs}, 4 \mathrm{H}), 7.91(\mathrm{~s}, 2 \mathrm{H}), 7.55\left(\mathrm{~d}, \mathrm{~J}_{3 \mathrm{H}, \mathrm{H}}=16.5 \mathrm{~Hz}\right), 7.54\left(\mathrm{~d}, 4 \mathrm{H}, \mathrm{J}_{3 \mathrm{H}, \mathrm{H}}=8.24 \mathrm{~Hz}\right), 7.45(\mathrm{~d}, 4 \mathrm{H}$, $\left.\mathrm{J}_{3 \mathrm{H}, \mathrm{H}}=5.5 \mathrm{~Hz}\right), 7.25(\mathrm{~m}, 6 \mathrm{H}), 2.99-2.90(\mathrm{~m}, 2 \mathrm{H}), 1.39\left(\mathrm{~d}, 12 \mathrm{H}, \mathrm{J}_{3 \mathrm{H}, \mathrm{H}}=6.9 \mathrm{~Hz}\right) .{ }^{13} \mathrm{C}$ NMR $(75.5$ $\left.\mathrm{MHz}, \mathrm{CDCl}_{3}\right): \delta=150.1,149.6,137.9,134.7,131.5,129.2,127.2,127.0,125.7,124.2,121.9$, 93.0, 92.4, 34.3, 24.3. Anal. Calcd.: C, 88.69; H, 6.38; Found: C, 88.41; H, 6.28

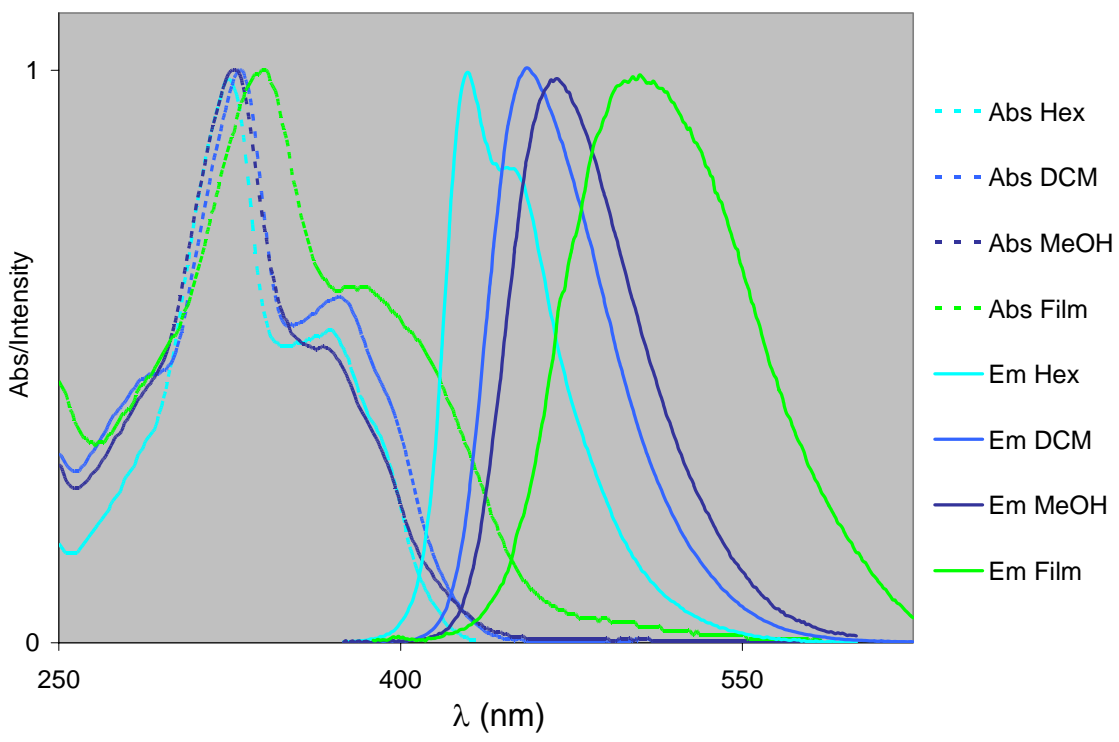

Figure 2. Absorption and emission spectra of $\mathbf{3}$ under different conditions. 
Table 2. Summary of optical data for compound 3.

\begin{tabular}{|c|c|c|c|c|}
\hline & Hexane & DCM & MeOH & Thin film \\
\hline $\begin{array}{c}\text { Absorbance } \\
\lambda_{\max }(\mathrm{nm})\end{array}$ & 324 & 328 & 329 & 341 \\
\hline $\begin{array}{c}\text { Emission } \\
\lambda_{\max }(\mathrm{nm})\end{array}$ & 430 & $\mathrm{E}=83,700 \mathrm{~cm}^{-1}$ & & \\
\hline & & 456 & 469 & 506 \\
\hline
\end{tabular}

Table 3. Preparation of Solutions for Sensing and Optical Measurements:

\begin{tabular}{|c|c|c|c|}
\hline Compound & Stock A & Stock B & Stock C \\
\hline \multirow{2}{*}{$\mathbf{2}$} & $10.0 \mathrm{mg} / 100 \mathrm{~mL}$ & & \\
& $100.0 \mathrm{mg} / \mathrm{L}$ & $10.0 \mathrm{mg} / \mathrm{L}$ & $2.00 \mathrm{mg} / \mathrm{L}$ \\
& $135 \mu \mathrm{M}$ & $13.5 \mu \mathrm{M}$ & $2.70 \mu \mathrm{M}$ \\
\hline \multirow{3}{*}{3} & $10.0 \mathrm{mg} / 100 \mathrm{~mL}$ & & \\
& $100.0 \mathrm{mg} / \mathrm{L}$ & $10.0 \mathrm{mg} / \mathrm{L}$ & $2.00 \mathrm{mg} / \mathrm{L}$ \\
& $176 \mu \mathrm{M}$ & $17.6 \mu \mathrm{M}$ & $3.51 \mu \mathrm{M}$ \\
\hline \multirow{2}{*}{4} & $10.0 \mathrm{mg} / 100 \mathrm{~mL}$ & $10.0 \mathrm{mg} / \mathrm{L}$ & $2.00 \mathrm{mg} / \mathrm{L}$ \\
& $100.0 \mathrm{mg} / \mathrm{L}$ & $13.5 \mu \mathrm{M}$ & $2.70 \mu \mathrm{M}$ \\
\hline
\end{tabular}

Table 4. Zinc triflate solutions:

Stock $\mathrm{D}=5.1 \mathrm{mg} / 500 \mathrm{~mL}=10.2 \mathrm{mg} / \mathrm{L}=28.1 \mu \mathrm{M}$

Stock $E=10.0 \mathrm{mg} / 500 \mathrm{~mL}=20.0 \mathrm{mg} / \mathrm{L}=55.0 \mu \mathrm{M}$

\begin{tabular}{|c|c|c|c|c|c|}
\hline Compound & $\begin{array}{c}\mathrm{mL} \text { of } \\
\text { Stock C }\end{array}$ & $\begin{array}{l}\text { Moles of } \\
\text { fluorophor }\end{array}$ & $\begin{array}{l}\text { Aliquots of } \\
\text { Stock D }\end{array}$ & $\begin{array}{c}\text { Moles of } \\
\mathrm{Zn}^{2+}\end{array}$ & $\begin{array}{l}\text { Equivalents } \\
\text { of } \mathrm{Zn}^{2+}\end{array}$ \\
\hline 2 & 3 & $8.1 \mathrm{nmol}$ & $x(100 \mu L)$ & $\mathrm{x}(2.81 \mathrm{nmol})$ & $\begin{array}{l}0.35 \\
0.69 \\
1.04 \\
1.38\end{array}$ \\
\hline 3 & 3 & $10.5 \mathrm{nmol}$ & $x(50 \mu \mathrm{L})$ & $\mathrm{x}(1.41 \mathrm{nmol})$ & $\begin{array}{c}0.13 \\
0.27 \\
0.40 \\
0.54 \\
0.67 \\
0.81 \\
0.94 \\
1.1 \\
1.2\end{array}$ \\
\hline 3 & 3 & $10.5 \mathrm{nmol}$ & $\begin{array}{l}0.5 \mathrm{~mL} \text { Stock } \\
\mathrm{D}+\mathrm{x}(100 \mu \mathrm{L})\end{array}$ & $\begin{array}{l}14.1 \mathrm{nmol}+ \\
\times(5.5 \mathrm{nmol})\end{array}$ & $\begin{array}{l}1.9 \\
2.4 \\
2.9 \\
3.4 \\
4.0\end{array}$ \\
\hline 4 & 3 & $8.1 \mathrm{nmol}$ & $x(75 \mu \mathrm{L})$ & $\mathrm{x}(2.21) \mathrm{nmol}$ & $\begin{array}{c}0.27 \\
0.55 \\
0.82 \\
1.1 \\
1.4\end{array}$ \\
\hline
\end{tabular}



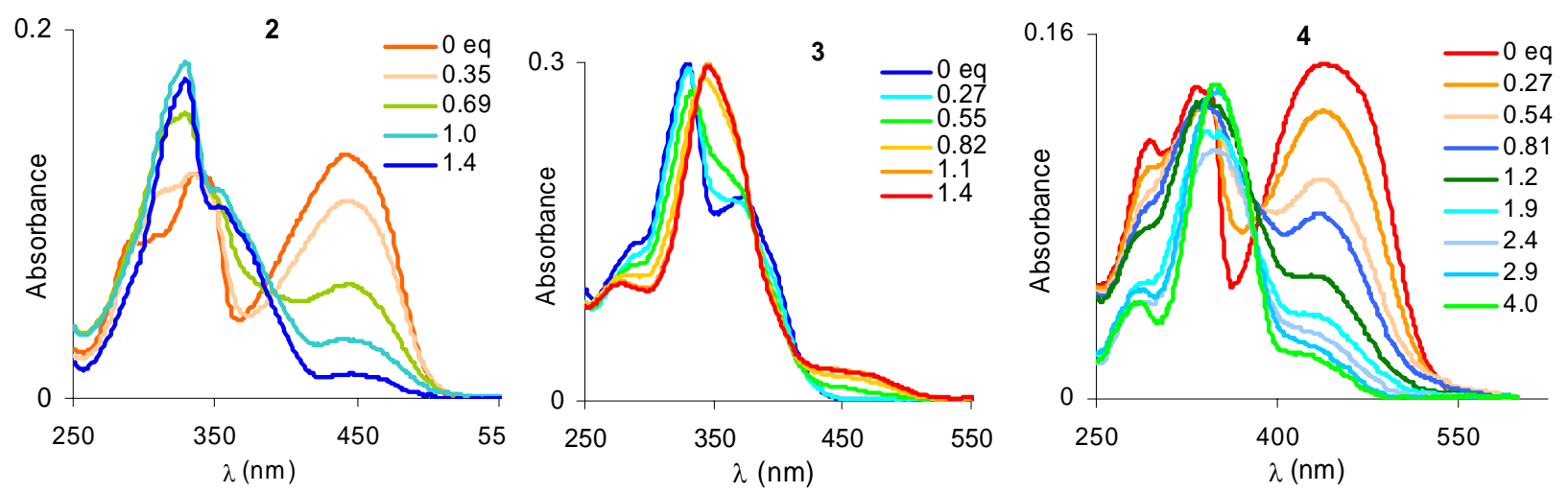

Figure 3. Absorption data: Addition of zinc triflate to 2-4.
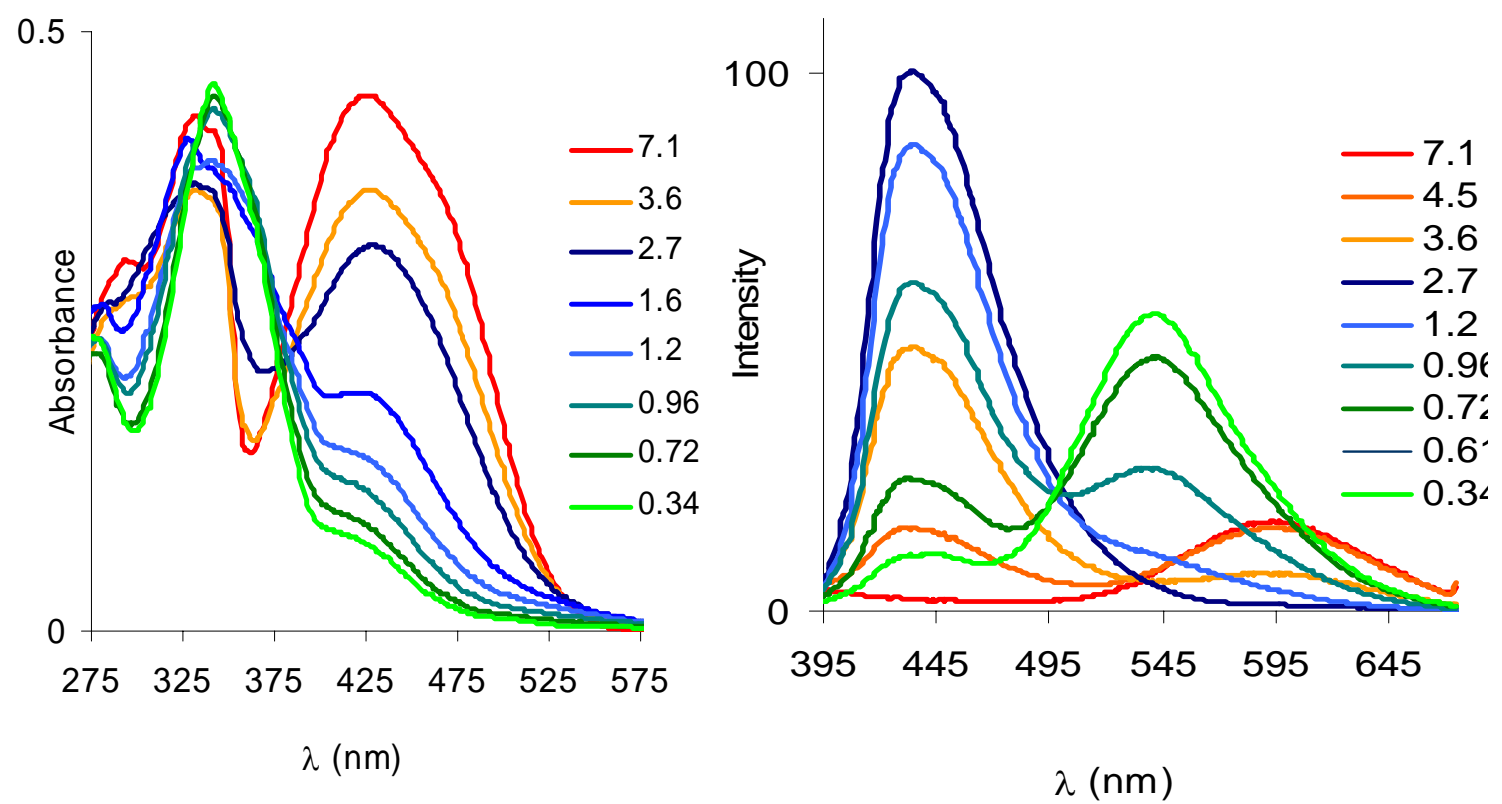

Figure 4. Addition of acid to 4 . Left is absorption, right is emission. The values at the side indicate the $\mathrm{pH}$ value of the solutions.

\section{References}

1) Wilson, J. N.; Josowicz, M.; Wang, Y. Q.; Bunz, U. H. F. Chem. Commun. 2003, 2962-2963, see supplementary material to that paper.

2) Wilson, J. N.; Windscheif, P. M.; Evan, U.; Myrick, M. L.; Bunz, U. H. F. Macromolecules 2002, 35, 8681-8683, see supplementary material to that paper. 